\title{
Comparison of the histopathology and prognosis of bilateral versus unilateral multifocal multicentric breast cancers
}

Hüseyin Kadioğlu ${ }^{*}$, Serdar Özbaş², Alper Akcan³, Aykut Soyder ${ }^{4}$, Lutfi Soylu², Savaş Koçak², N Zafer Cantürk ${ }^{5}$, Mustafa Tükenmez ${ }^{6}$ and Mahmut Müslümanoğlu

\begin{abstract}
Background: Multiple breast cancers may present with different clinical and biological characteristics. The data indicate that multifocal (MF), multicentric (MC), and bilateral synchronous (BS) breast cancers (BC) are more aggressive and have an equivalent or moderately poorer survival rate compared with unilateral cases. However, a comparison of these multiple breast cancers has not been covered in the literature. The aim of this study was to describe the histopathological characteristics of patients suffering from MF, MC, and BS breast carcinoma and to compare their prognoses.
\end{abstract}

Methods: Retrospective data for MF, MC, and BS breast carcinoma patients treated in five different breast cancer units in Turkey between 2003 and 2012 were collected. MF and MC cancers were defined as more than one lesion in the same quadrant or in separate quadrants, respectively.

Results: There were 507 patients (271 MF, 147 MC, and 89 BS) treated in this time period. BS breast carcinoma patients were younger than the other groups $(44.83 \pm 9.6,47.27 \pm 11.6$, and $51.11 \pm 11.8$ years for $B S, M F$, and MC breast carcinoma patients, respectively). MFBC and MCBC patients in this study were younger than the ages reported in Western literature, but this result was similar to the ages reported in Eastern literature. The five-year survival rates and recurrence rates were not statistically different among groups $(P=0.996$ and $P=0.263$, respectively). According to univariate analyses, tumor size, histological grade, and lymph node status were statistically significant factors that affected survival. However, only lymph node involvement was significant for survival according to multivariate analyses.

Conclusions: The clinical significance of MF, MC, and BS breast cancers is still unclear and their influence on prognosis is controversial. Disease-free and overall survival rates of BS breast cancers might be similar to MF and MC breast cancers.

Keywords: Bilateral synchronous, Multifocal, Multicentric, Breast cancer

\section{Background}

Unilateral, multifocal, and multicentric breast cancers have been of interest to oncology professionals for many years, but their impact on prognosis and survival is controversial [1]. While early clinical trials advocated mastectomy for multifocal and multicentric tumors [2], the

\footnotetext{
* Correspondence: huseyinkadioglu@gmail.com

'Department of General Surgery, Bezmialem Vakif University, Istanbul Adnan Menderes Bulvarı Vatan Caddesi, 34093 Fatih/Istanbul, Turkey

Full list of author information is available at the end of the article
}

recent literature suggests local recurrence rates do not differ from unifocal tumors [3].

Bilateral synchronous breast cancer (BSBC) is a rare entity with an incidence of between 1 and 3\%. Surprisingly, there has been no increase in BSBC incidence since the 1980s [4]. However, its incidence has been reported to be as high as $12 \%$ [5]. Different time intervals have been used to define BSBC. In 1921, Kilgore defined BSBC as a breast cancer in which both tumors are diagnosed at the same time [6]. Since 1921, different time intervals have been used ranging from one month to five 
years [7]. A widely accepted definition of BSBC is that of Hartman et al., who defined BSBC in 2007 as a tumor diagnosed within 90 days of the first tumor [4].

Another dilemma regarding BSBC is the relationship between tumors. Contradictory results on BSBC have been published. Some authors have demonstrated similarity in histologic subtype [8], tumor grade [9], and hormone receptor status [10] between tumors, suggesting a single-cell origin, but other authors have reported different results [11]. Some authors believe that the tumors are genetically different [12].

The survival effect of BSBC is also a dilemma for oncology professionals. While some reports advocate a worse prognosis for $\mathrm{BSBC}$, other reports do not suggest a worse prognosis for $\mathrm{BSBC}$.

According to the current literature, multifocal breast cancer (MFBC) does not affect survival. Recent studies have compared BSBCs with unilateral breast cancer, unifocal breast cancer, MFBC, and multicentric breast cancer $(\mathrm{MCBC})$. We believe that this heterogeneity is the cause of the differences reported in survival ratios, and that tumors beginning from more than one focus should be compared with other tumors beginning from more than one focus.

The aim of this study was to compare the histopathological findings and survival of BSBC, MFBC, and MCBC cases and to analyze the effect of molecular subtype on survival.

\section{Methods}

The retrospective data for BSBC, MFBC, and MCBC patients treated in five different breast centers in Turkey between 2003 and 2012 were collected.

All of these centers are university hospitals and have a similar follow-up schedule for breast cancer based on national comprehensive cancer network $(\mathrm{NCCN})$ guidelines. The data were obtained from the oncology departments' follow-up files of the patients.

The following patients were excluded from the study: patients who were treated with neo-adjuvant chemotherapy, patients who had a history of contralateral breast cancer, patients who received chemotherapy or radiotherapy for any kind of malignancy, patients who were male; patients who were lost at follow-up, and patients who were not seen by a medical professional in the previous six months.

Patients diagnosed with contralateral breast cancer in the most recent 90 days were defined as BSBC. MFBC was defined as tumors separated by normal breast tissue in the same quadrant, and MCBC was defined as tumors separated by normal breast tissue in different quadrants. Multifocal and multicentric tumors were identified by macroscopic examination of pathology specimens. In situ components of the tumors were not added to the tumor size. Tumors connected by in situ cancer were defined as asunder foci. Also tumors which are closer than $5 \mathrm{~mm}$ to each other are defined as satellite nodules.

A total of 507 patients met these criteria. Age at diagnosis, menopausal status, number of tumor foci (index side for BSBC), tumor size (index side for BSBC), histological type (index side for $\mathrm{BSBC}$ ), histological grade (HG; defined using the Modified Bloom-Richardson grading system), lymphovascular invasion (LVI) status, lymph node involvement, estrogen receptor status, progesterone receptor status, HER2/neu status, stage, and molecular subtype were recorded.

All five centers were using ultrasonography for the preoperative evaluation of the axillary, supra-clavicle, and internal mammary lymph nodes. All five centers were using immunohistochemical methods to define HER2/neu status up to year 2009; in situ hybridization techniques have been in use since year 2009 .

Estrogen receptor-positive patients with low Ki 67 levels (<14\%), HER2/neu-negative, and low-grade tumors were defined as luminal A. Estrogen receptor-negative and/or patients with high Ki 67 levels (>20\%) and HER2/ neu-negative tumors were defined as luminal B. HER2/ neu-positive patients were defined as HER2 type. Estrogen receptor-, progesterone receptor-, and HER2/neu-negative tumors were defined as triple negative. The histopathological parameters of the index side were recorded for $\mathrm{BSBC}$, and the type of the surgery was recorded as mastectomy in the BSBC group if it was applied to any side. The local recurrence and metastatic status at the final follow-up were also recorded.

\section{Statistical analysis}

The frequency and descriptive analyses of the cases were recorded. The qualitative data were analyzed using Fisher's exact test, and the quantitative data were analyzed using the Mann-Whitney U test. Survival analyses were conducted using the Kaplan Meier test and the Cox multivariate regression test, and logistic regression tests were used for subgroup analyses. The data analysis was performed using SPSS software (SPSS Statistics for Windows, Version 17.0. Chicago: SPSS Inc).

\section{Results}

The total number of patients included in the study was 507. There were 89 patients in the BSBC group, 271 patients in the MFBC group, and 147 patients in the MCBC group. Surprisingly, the MCBC patients were significantly older than the other two groups. The mean age in the MCBC group was $51.11 \pm 11.9$ years (range of 29 to 83 years). The mean ages of the MFBC and BSBC groups were $47.27 \pm 11.63$ years (range of 29 to 84 years) and $44.83 \pm 9.67$ years (range of 27 to 84 years), 
respectively $(P<0.001)$. The majority of the MCBC patients were postmenopausal $(P<0.001)$.

The BSBC cases had more foci than the MFBC and MCBC cases (mean focus number of $3.12 \pm 1.46,2.88 \pm$ 1.54 , and $2.3 \pm 0.77$, respectively; $P<0.001$ ). In addition, the mean tumor size was larger in the BSBC patients compared to that in the MFBC and MCBC patients $(55.88 \pm 23.7 \mathrm{~mm}, 22.09 \pm 12.0 \mathrm{~mm}$, and $34.96 \pm 20.5 \mathrm{~mm}$, respectively; $P<0.001)$. The most common histological type in all groups was invasive ductal carcinoma $(P=0.001)$.

Compared to the other groups, the histological grade was higher in the MCBC group $(P<0.001)$, and the majority of the patients were lymphatic vascular invasionpositive in the BSBC group $(P<0.001)$.

There were no NO patients in the BSBC group, and most of the N3 patients were in the BSBC group. This difference was statistically significant $(P<0.001)$. A total of $13 \mathrm{~N}$-positive patients did not undergo axillary surgery whilst their sentinel lymph node biopsy was positive; all 13 patients underwent additional axillary radiotherapy. No local recurrences occurred in these patients.

Estrogen receptor status and progesterone receptor status were also similar among the groups $(P=0.515$ and $P=0.193$, respectively). Compared to the other patient groups, there were more HER2/neu-positive tumors in the MCBC patient group $(P=0.006)$.

Whilst we cannot obtain the detailed treatment regimens we reported the obtainable treatment regimens for three groups (89 patients $(100 \%)$ for the BSBC group, 230 patients $(84.8 \%)$ for the MFBC group and 100 patients for the MCBC group). Due to the heterogeneity we did not apply statistical analyses.

The median follow-up time was $46.42 \pm 23.2$ months (range of 1 to 108 months). However, for each group the median follow-up was $49.63 \pm 23.98,44.29 \pm 22.85$, and $40.15 \pm 19.92$ months for MFBC, MCBC, and BSBC groups, respectively.

Local recurrence and mortality were not different among the groups ( $P=0.263$ and $P=0.996$, respectively). Five local recurrences occurred in BSBC group, three of which were breast cancer recurrences and two of which were axillary cancer recurrences. A total of 14 local recurrences occurred in the MFBC group, eight of which were breast cancer recurrences and six of which were axillary cancer recurrences. Three local recurrences occurred in MCBC group, two of which were breast cancer recurrences and one of which was axillary cancer recurrence. Comparative analyses of these data are shown in Table 1.

Local recurrence rates dependent on surgery type were analyzed (5.6\% for MFBC, $2.0 \%$ for MCBC, and 5.2\% for $\mathrm{BSBC}$ ), and there was no significant difference between surgery types ( $95.0 \mathrm{CI}$ of 98.5 to $104.25 ; P=0.369$ ). These low local recurrence rates could be explained by careful selection of patients for breast-conserving surgery. A trustable clean surgical margin was defined as at least $2 \mathrm{~mm}$ at all five centers, and re-excisions were made toward reaching these margins. Otherwise, a mastectomy was performed.

Univariate analyses of survival indicated significant differences in relation to histological type $(P<0.001)$, histological grade $(P=0.024)$, estrogen receptor status $(P<0.001)$, progesterone receptor status $(P<0.001)$, lymph node involvement $(P=0.025)$, and molecular subtype $(P<0.001)$. However, BSBC diagnosis, menopausal status, lymphatic-vascular invasion, and HER2/neu status were not statistically significant $(P=0.544, P=0.092, P=0.875$, and $P=0.104$, respectively). Having a mastectomy was associated with a poorer prognosis $(P<0.001)$.

There were not any statistically significant differences in overall survival rates (the five-year survival rate was $82.7 \%, 86.9 \%$, and $90.5 \%$ for the BSBC, MFBC, and MCBC groups, respectively; $P=0.416$ ) (Figure 1 ). The five-year disease-free survival rate was $82.7 \%, 80.7 \%$, and $75.5 \%$ for the $\mathrm{BSBC}, \mathrm{MFBC}$, and $\mathrm{MCBC}$ groups, respectively $(P=$ 0.024). When we compared the stage-dependent survival analyses of the three groups, there was no significant difference among the mortality rates $(95.0 \mathrm{CI}$ of 0.731 to 15.191, $P=0.87$; $95.0 \mathrm{CI}$ of 0.661 to $5.240, \mathrm{P}=0.73$; and $95.0 \mathrm{CI}$ of 0.106 to $1.452, P=0.63$ for Stage I, II, and III, respectively; Figure 2a, b, c).

Metastasis rates were different between the groups. The highest ratio was in the MFBC group (18.4\%, 27 out of 271 patients) and the lowest ratio was in the BSBC group ( $9 \%, 8$ out of 89 patients, $P=0.04$ ). We believe that these ratios are the result of our small sampling size and that this caused a statistical bias regarding the metastatic ratios of this study.

We analyzed the usual prognostic parameters that were statistically significant in Kaplan-Meier analyses using the Cox multivariate regression test. Only lymph node status, lymphatic-vascular invasion, and estrogen receptor status had a statistically significant effect on survival $(P=0.006$, $P=0.027$, and $P=0.41$, respectively) (Table 2). Progesterone receptor status alone did not have a statistically significant effect on survival. Table 3 shows the univariate and multivariate analyses data for the prognostic parameters.

Finally, we analyzed the survival effect of molecular subtypes as luminal A, luminal B, triple negative, and HER2 type. While no survival difference was found between luminal A and B patients $(P=0.142)$, mortality in triplenegative patients was 5.11-fold greater than in luminal A patients (95.0\% CI of 2.06 to $9.48, P<0.001$; Table 3 ).

\section{Discussion}

Multiple simultaneous tumor foci in the same breast are referred to as multifocal or multicentric, but there is no 
Table 1 Analyses of histopathological parameters and survival between the groups

\begin{tabular}{|c|c|c|c|c|}
\hline & $\begin{array}{c}\text { BSBC } \\
(n=89)\end{array}$ & $\begin{array}{c}\text { MFBC } \\
(n=271)\end{array}$ & $\begin{array}{c}\text { MCBC } \\
(n=147)\end{array}$ & $P$ \\
\hline Age & $44.83 \pm 9.67(27-84)$ years & $47.27 \pm 11.63(19-84)$ years & $51.11 \pm 11.94(29-83)$ years & $<0.001$ \\
\hline Menopause Status & & & & $<0.001$ \\
\hline Premenopausal & $56(62.9 \%)$ & $168(62.0 \%)$ & $60(40.8 \%)$ & \\
\hline Postmenopausal & $33(37.1 \%)$ & $103(38.0 \%)$ & $87(59.2 \%)$ & \\
\hline Number of tumors (Index side for BSBC) & $3.12 \pm 1.46$ & $2.88 \pm 1.54$ & $2.3 \pm 0.77$ & $<0.001$ \\
\hline Tumor size (mm) (Index side for BSBC) & $55.88 \pm 23.7$ & $22.0 \pm 12.0$ & $34.9 \pm 20.5$ & $<0.001$ \\
\hline Histological type & & & & 0.001 \\
\hline Invasive ductal & $75(84.2 \%)$ & $198(73 \%)$ & $134(91.1 \%)$ & \\
\hline Mix & $5(5.6 \%)$ & $23(8.4 \%)$ & $9(6.1 \%)$ & \\
\hline Invasive lobular & $6(6.7 \%)$ & $33(12.1 \%)$ & $4(2.7 \%)$ & \\
\hline Others & $3(3.3 \%)$ & $14(6.1 \%)$ & 0 & \\
\hline Histological Grade (Modified Bloom Richardson) & & & & $<0.001$ \\
\hline I & $10(11.5 \%)$ & $40(15.0 \%)$ & $34(23.1 \%)$ & \\
\hline$\|$ & $66(75.9 \%)$ & $113(42.3 \%)$ & $36(24.5 \%)$ & \\
\hline III & $11(12.6 \%)$ & $114(42.7 \%)$ & $77(52.4 \%)$ & \\
\hline Lymphatic vascular invasion & & & & $<0.001$ \\
\hline Positive & $68(76.4 \%)$ & $134(49.4 \%)$ & $77(42.5 \%)$ & \\
\hline Negative & $21(23.6 \%)$ & $137(50.6 \%)$ & $70(47.6 \%)$ & \\
\hline Lymph node involvement & & & & $<0.001$ \\
\hline No & 0 & $128(47.2 \%)$ & $68(46.3 \%)$ & \\
\hline N1 & $17(19.1 \%)$ & $83(30.6 \%)$ & $17(11.6 \%)$ & \\
\hline N2 & $41(46.1 \%)$ & $40(14.8 \%)$ & $34(23.1 \%)$ & \\
\hline N3 & $31(34.8 \%)$ & $20(7.4 \%)$ & $28(19.0 \%)$ & \\
\hline Estrogen receptor status & & & & 0.515 \\
\hline Positive & $53(59.6 \%)$ & $147(54.8 \%)$ & $87(59.2 \%)$ & \\
\hline Negative & $36(40.4 \%)$ & $124(45.8 \%)$ & $60(40.8 \%)$ & \\
\hline Progesterone receptor status & & & & 0.193 \\
\hline Positive & $69(77.5 \%)$ & $195(72.0 \%)$ & $98(66.7 \%)$ & \\
\hline Negative & $20(22.5 \%)$ & $76(28.0 \%)$ & $49(33.3 \%)$ & \\
\hline HER2/neu status & & & & 0.02 \\
\hline Positive & $8(9.0 \%)$ & $16(5.9 \%)$ & $24(14.2 \%)$ & \\
\hline Negative & $73(90.1 \%)$ & $255(94.1 \%)$ & $123(83.7 \%)$ & \\
\hline Molecular subtypes & & & & 0.03 \\
\hline Luminal A & $46(51.7 \%)$ & $135(49.82 \%)$ & $69(46.9 \%)$ & \\
\hline Luminal B & 16 (18.08\%) & $51(18.8 \%)$ & 19 (12.9\%) & \\
\hline Triple negative & 19 (21.3\%) & $69(25.5 \%)$ & $35(23.8 \%)$ & \\
\hline HER2 type & $8(9.0 \%)$ & $16(5.9 \%)$ & $24(16.3 \%)$ & \\
\hline Type of surgery & & & & $<0,001$ \\
\hline $\mathrm{BCS}+\mathrm{SLN}$ & 0 & 132 (48.7\%) & $32(21.8 \%)$ & \\
\hline$B C S+A D$ & $23(25.8 \%)$ & 105 (38.7\%) & $4(2.7 \%)$ & \\
\hline $\mathrm{MST}+\mathrm{SLN}$ & $13(14.6 \%)$ & $11(4.1 \%)$ & 30 (20.4\%) & \\
\hline MRM & $53(59.6 \%)$ & $23(8.5 \%)$ & 81 (55.1\%) & \\
\hline
\end{tabular}


Table 1 Analyses of histopathological parameters and survival between the groups (Continued)

\begin{tabular}{cccc}
\hline Adjuvant treatment modalities & & Not applied \\
Chemotherapy & $87(97.7 \%)$ & $141(52.0 \%)$ & $80(54.4 \%)$ \\
Radiotherapy & $89(100 \%)$ & $225(83.0 \%)$ & $75(51.0 \%)$ \\
Endocrine therapy & $60(67.4 \%)$ & $151(55.7 \%)$ & $85(57.8 \%)$ \\
Targeted therapy & $8(9.0 \%)$ & $12(4.4 \%)$ & $21(14.2 \%)$ \\
Local recurrence & $5(5.6 \%)$ & $14(5.2 \%)$ & $3(2.0 \%)$ \\
Mortality & $7(7.9 \%)$ & $22(8.1 \%)$ & $12(8.2 \%)$ \\
\hline
\end{tabular}

AD: Axillary dissection; BCS: Breast conserving surgery; MRM: Modified radical mastectomy; MST: Mastectomy; SLN: Sentinel lymph node biopsy.

consensus on the terminology [13]. Classically, tumors in different quadrants are referred to as multicentric. Pathologists define multiple simultaneous primary lesions when there are two or more tumor foci without intervening malignant tissue [14]. Pathologists define tumors as multifocal when only one breast quadrant is involved, and they define tumors as multicentric when two or more quadrants are involved [14]. Radiologists do not have an exact definition, but tumors are usually considered multifocal when the distance between the tumors is less than or equal to $5 \mathrm{~cm}$, and are considered multicentric when the distance between the tumors is more than $5 \mathrm{~cm}$ [15]. The estimated prevalence differs from 4 to $65 \%$, and this variability is mainly due to the lack of standardization in the gross examination and sampling of breast specimens [16].
BSBC is still a subject of debate for oncology professionals. The data regarding BSBC are sophisticated, and the study results are confusing. We believe that the patient choice in the studies is the cause of this confusing status. Synchronous and metachronous tumors are all discussed similarly in these studies.

In addition, the comparisons of the groups are heterogeneous. Tumors that begin from more than one focus (such as BSBC) are compared with unilateral and unifocal tumors. At the same time, the biology of the tumors beginning from more than one focus should be different.

The literature reports are not all based on the same definition of BSBC [17]. A recent study evaluating 5292 patients in Germany found that differences in outcome between synchronous and metachronous cancers depend on time interval and that the optimal cut-off for the

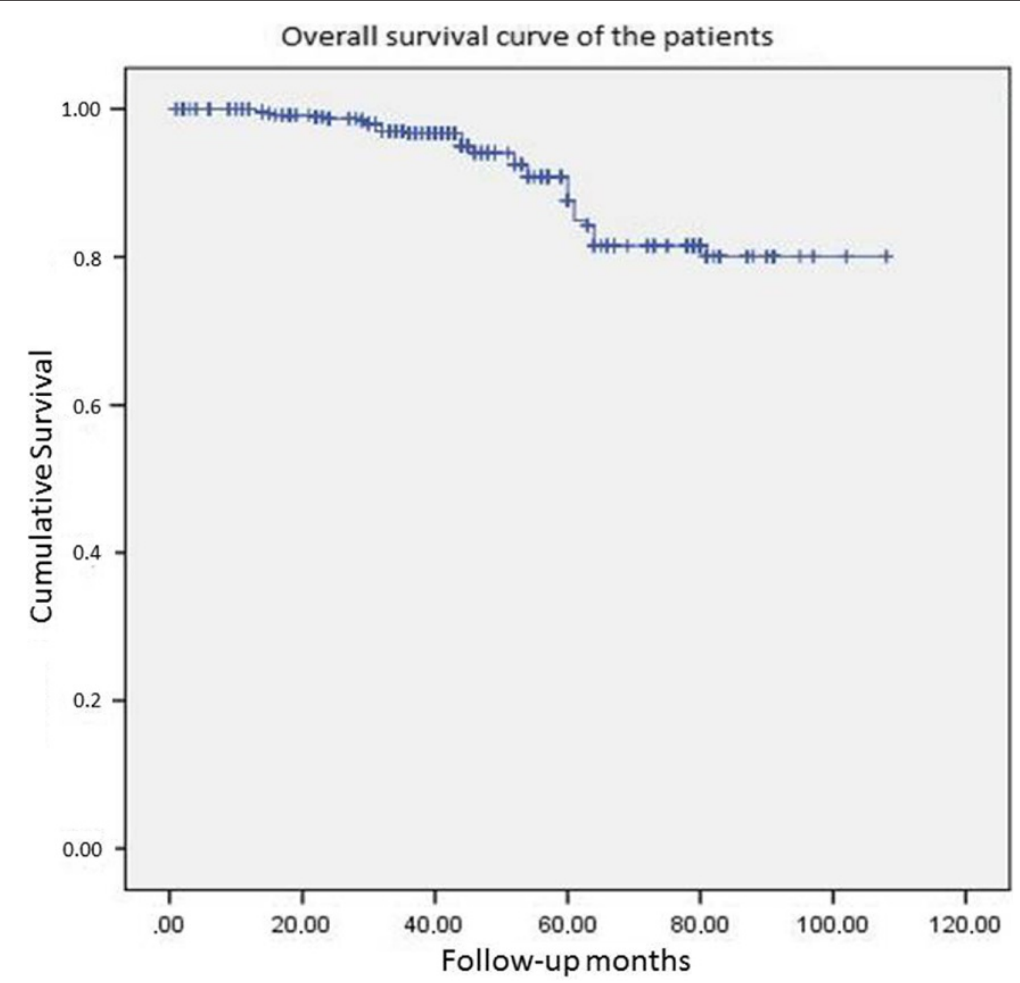

Figure 1 Overall survival curve. 


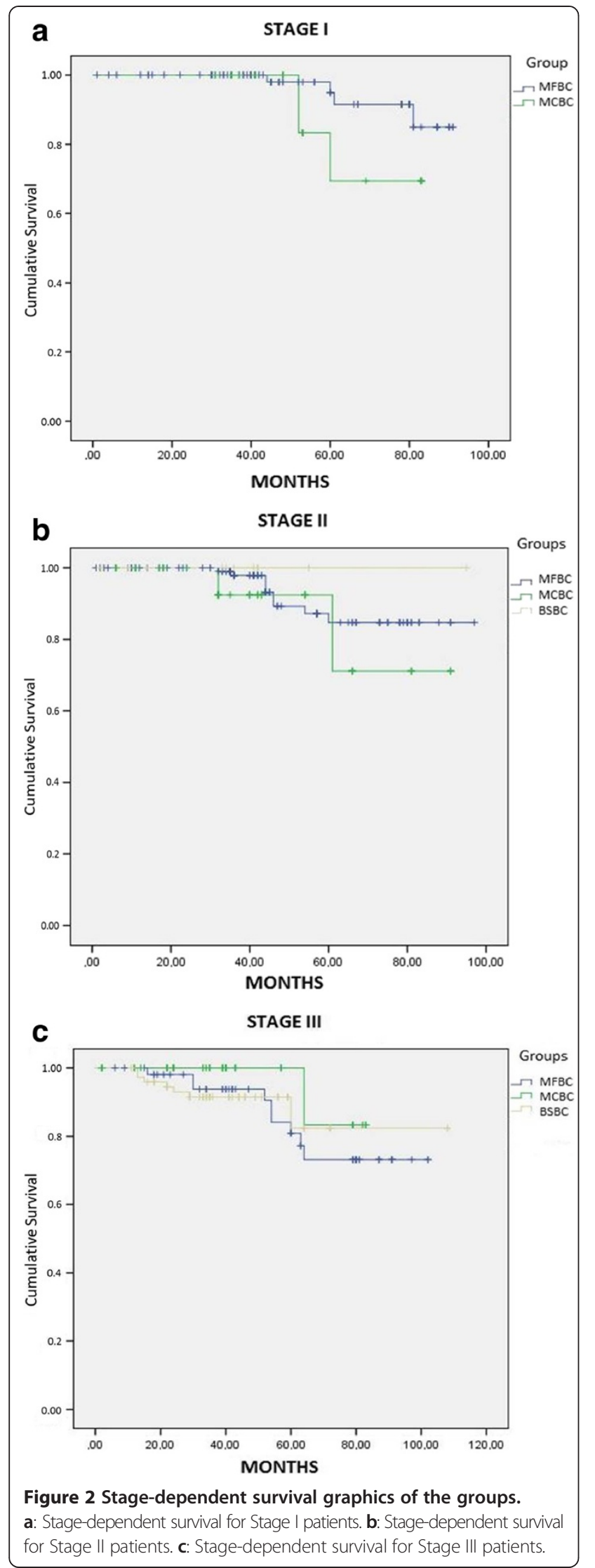

Table 2 Multivariate analyses for the effect of classical histopathological parameters on overall survival between the groups

\begin{tabular}{cccc}
\hline & $\boldsymbol{P}$ & $\mathbf{9 5 \%} \mathbf{C l}$ & $\mathbf{O R}$ \\
\hline Histological grade & 0.027 & $0.198-0.905$ & 4.913 \\
Estrogen receptor status & 0.41 & $0.116-0.954$ & 4.190 \\
Progesterone receptor status & 0.171 & - & - \\
Lymph node involvement & 0.006 & $1.396-7.165$ & 7.614 \\
Molecular subtypes & $<0.001$ & $2.74-13.55$ & 23.63 \\
\hline
\end{tabular}

Cl: Confidence Interval; OR: Odds Ratio.

overall survival of BSBC is 4.5 months [18]. We believe the optimal definition of BSBC is cancers diagnosed simultaneously in both breasts or within three months (90 days) of diagnosis of the first tumor as described by Hartman et al. [4]. For these reasons, it is difficult to compare our results with the literature. We compared our results with recent literature in which results for BSBC were separately given for at least for 40 patients, and the definition of BSBC was similar to ours. Only a few studies have independently compared synchronous and metachronous breast cancers, and these studies have contradictory results $[4,19-21]$.

Roder et al. concluded that the risk for BSBC increases with age [21]. In general, other studies report a median age older than 50 years [22]. A significant portion of our BSBC patients were premenopausal $(62.9 \%)$ with a median age that was younger ( $44.83 \pm 9.67$ years) than other Western studies, yet the median age was similar to that reported by Shi et al. (BSBC patient median age of $49 \pm$ 15 years) [19]. Similarly, our MFBC and MCBC patients were younger (the median age for MFBC and MCBC was $47.27 \pm 11.63$ and $51.11 \pm 11.94$ years, respectively) than the ages reported in Western literature, but this result was similar to the ages reported in Eastern literature [23]. Recent literature evaluating 1492 consecutive breast

Table 3 Univariate analyses for the effect of classical histopathological parameters on overall survival between the groups

\begin{tabular}{cc}
\hline & $\boldsymbol{P}$ \\
\hline Overall survival between groups & 0.544 \\
Menopause status between groups & 0.092 \\
Histological type & 0.488 \\
Histological grade & 0.024 \\
Lymphovascular invasion & 0.875 \\
Estrogen receptor status & $<0.001$ \\
Progesterone receptor status & $<0.001$ \\
HER2/neu status & 0.104 \\
Lymph node involvement & 0.045 \\
Molecular subtypes & $<0.001$ \\
\hline
\end{tabular}


cancer patients from Turkey has shown that $41.2 \%$ of the patients are younger than 50 years [24]. Furthermore, another study from Turkey evaluating MFBC and MCBC has reported that $51.4 \%$ of the patients are premenopausal [25]. These data suggest different biological behaviors of breast cancer in different races.

In our study, the mean tumor size was larger than reported in the literature for BSBC [19-22], yet all of these reported studies included patients with neo-adjuvant chemotherapy. Only one study from Italy excluded patients with neo-adjuvant chemotherapy, but the mean tumor size in our study was larger than that reported by Intra et al. [24].

The incidence of lobular histology is between 15 and $22 \%$ for BSBC in Western studies [20,22,26], but the incidence was $6.7 \%$ in our study, which was similar to the results reported by Eastern studies [23,25]. A large study from British Columbia evaluating 25,320 breast cancer patients reported a lobular histology incidence of $13.6 \%$ [27]. The result for histological type was $12 \%$ in the study of Cabioglu et al. [28]. This percentage is slightly less than the ratio from British Columbia. Our cumulative ratio for $\mathrm{MFBC}$ and $\mathrm{MCBC}$ was $9.6 \%$, and this ratio was slightly lower than both studies, but concordant with Eastern studies [23].

A substantial percentage of the tumors had high histological grades. This result was similar to the literature in which a histological grade of II or III was assigned to between 33.0 and $79.5 \%$ of BSBC patients and between 85.6 and $90.8 \%$ of MFBC and MCBC patients [20,25]. However, no studies are available that compare histological grade in BSBC to that in MCBC and MFBC.

As confirmed by NCCN guidelines, a higher histological grade is an unfavorable factor for breast cancer. Thus, we analyzed the survival effect of histological grade by univariate and multivariate analyses. Histological grade had a negative effect on survival in both analyses (univariate analysis: $P=0.024$; multivariate analysis: $95 \% \mathrm{CI}$ of 0.198 to $0.905, P=0.027$ ).

The impact on the survival of these patients is a matter of discussion. Some publications conclude that BSBC patients have worse prognoses $[20,22,26]$, and other reports do not refer to significant differences between unilateral and BSBC [26]. Indeed, the largest study of BSBC is a $\mathrm{PhD}$ thesis from Adelaide University (Australia) [20]. McCaul [20] analyzed 4424 women with BSBC who were diagnosed in the same month, and the results of his thesis suggest that tumor burden should be taken into consideration. He reported that bilateral stage I tumors have a similar prognosis when compared with unilateral stage I tumors, but that the survival of bilateral stage II tumors is worse than unilateral stage II tumors. However, a survival comparison of one side stage II and one side stage I tumors with unilateral stage II tumors showed that BSBC patients have a similar survival rate. In this study, we could not find a statistically significant difference in the five-year overall survival, but the fiveyear survival rates were lower for the BSBC group compared to the other groups. Perhaps a new classification in which the tumor burden of both breasts is included should be made.

Gene expression profiling has led to a new molecular classification of breast cancer with the triple-negative subgroup having the worse prognosis. As predicted, the triple-negative group had the worse prognosis in this study.

The ratio of HER2/neu positivity is smaller than the literature in the results of this study. We believe that the cause of this difference could be the small sampling size.

Thus imaging modalities become more important at the decision of BSBC-MFBC and $\mathrm{MCBC}$, the role of magnetic resonance imaging (MRI) is another redundant point for the oncology specialists. Recent literature about the role of imaging modalities in the diagnosis of BSBC suggests that a family history of breast cancer, a multifocal breast tumor, or the presence of an invasive lobular carcinoma should be arguments for the realization of a breast MRI to eliminate contralateral malignancy [29]. Another prospective study from Turkey that explores the utility of positron emission tomography/ computed tomography (PET/CT) scans to assess tumor multifocality and multicentricity suggests that: "However, the specificity of the MR is rather poor, being less than half that of PET/CT scans". Thus PET/CT scan data should be considered before aggressive surgery is scheduled [30].

Only two case studies evaluating BSBC patients from Turkey have been published (with a total of 20 patients) $[31,32]$. However, larger studies evaluating multifocal and multicentric breast cancers in Turkey are available $[25,28]$. The number of patients reported in this study is the largest series in Turkey. It is important to report the status of BSBC patients in Turkey.

In conclusion, we need a common and accepted definition for BSBC. Prospective studies that include patients who fit this description should be conducted to determine the survival statistics for BSBC. Staging of BSBC should be investigated, and tumor burden should also be taken into account.

The data for treatment regimens were insufficient, which may affect the results of this study. In addition, the follow-up period was too short, and long-term results could be different. Thus, this study can only give a preliminary hypothesis regarding the short-term prognosis for tumors with multiple foci.

Of course the major limitation of this study is the fact that it is a retrospective analysis, as well as the known limitations and biases of the multicentric studies. 


\section{Conclusions}

In conclusion $\mathrm{BSBC}, \mathrm{MFBC}$, and $\mathrm{MCBC}$ had similar survival rates in this study. The multivariate analyses identified molecular subtypes and lymph node status as the main factors that affected survival. However, results from the literature are confusing because there are different definitions and different survival results. Thus, a decision about BSBC survival rates can be made only after a large trial with a standardized definition of BSBC is conducted.

\begin{abstract}
Abbreviations
AD: Axillary dissection; BS: bilateral synchronous; BSBC: Bilateral synchronous breast cancer; BC: breast cancers; BCS: Breast conserving surgery; Cl: Confidence Interval; HG: histological grade; LVI: Iymphovascular invasion; MST: Mastectomy; MRM: Modified radical mastectomy; MC: multicentric; MCBC: multicentric breast cancer; MF: multifocal; MFBC: multifocal breast cancer; NCCN: national comprehensive cancer network; OR: Odds Ratio.
\end{abstract}

\section{Competing interests}

The authors declare that they have no conflicts of interest.

\section{Authors' contributions}

HK, SÖ: Manuscript writing, data collection, design of the study. AA, AS, LS, SK, N ZC, MT: Data collection. MM, SK, SÖ: Critical Review. All authors read and approved the final manuscript.

\section{Acknowledgements}

None.

\section{Author details}

${ }^{1}$ Department of General Surgery, Bezmialem Vakif University, Istanbul Adnan Menderes Bulvarı Vatan Caddesi, 34093 Fatih/Istanbul, Turkey. ${ }^{2}$ Department of General Surgery, Güven Hospital, Şimşek Sok. No:29 06540 Kavaklıdere, Ankara, Turkey. ${ }^{3}$ Department of General Surgery, Erciyes University, Erciyes Üniversitesi Tıp Fakültesi Dekanlığı, 38039 Talas/Kayseri, Turkey. ${ }^{4}$ Department of General Surgery, Adnan Menderes University, 09100 Aydın, Turkey. ${ }^{5}$ Department of General Surgery, Kocaeli University, 41380 Umuttepe/ KOCAELI, Turkey. ${ }^{6}$ Department of General Surgery, Istanbul University Medical Faculty, 34083 Istanbul, Turkey.

\section{Received: 7 October 2013 Accepted: 6 August 2014}

Published: 20 August 2014

\section{References}

1. Fisher ER, Sass R, Fisher B, Gregorio R, Brown R, Wickerham L: Pathologic findings from the National Surgical Adjuvant Breast Project (protocol 6). II. Relation of local breast recurrence to multicentricity. Cancer 1986, 57:1717-1724.

2. Kurtz JM, Jacquemier J, Amalric R, Brandone H, Ayme Y, Hans D, Bressac C, Spitalier JM: Breast conserving therapy for macroscopically multiple cancers. Ann Surg 1990, 212:38-44.

3. Gentilini O1, Botteri E, Rotmensz N, Da Lima L, Caliskan M, Garcia-Etienne CA, Sosnovskikh I, Intra M, Mazzarol G, Musmeci S, Veronesi P, Galimberti V, Luini A, Viale G, Goldhirsch A, Veronesi U: Conservative surgery in patients with multifocal/multicentric breast cancer. Breast Cancer Res Treat 2009, 113:577-583.

4. Hartman M, Czene K, Reilly M, Adolfsson J, Bergh J, Adami HO, Dickman PW, Hall P: Incidence and prognosis of synchronous and metachronous bilateral breast cancer. JCO 2007, 4210-4216.

5. Pomerantz RA, Murad T, Hines JR: Bilateral breast cancer. Am Surg 1989, 55:441-444.

6. Kilgore AR: The incidence of cancer in the second breast. JAMA 1921, 77:454-457.

7. Haagensen CD: Diseases of the Breast. 2nd edition. Philadelphia: Saunders; 1971.

8. Burns PE, Dabbs K, May C, Lees AW, Birkett LR, Jenkins HJ, Hanson J: Bilateral breast cancer in northern Alberta: risk factors and survival patterns. Can Med Assoc J 1984, 130:881-886.
9. Sterns EE, Fletcher WA: Bilateral cancer of the breast: a review of clinical, histologic, and immunohistologic characteristics. Surgery 1991, 110:617-622.

10. Dawson PJ, Maloney T, Gimotty P, Juneau P, Ownby H, Wolman SR: Bilateral breast cancer; one disease or two? Breast Cancer Res Treat 1991, 19:233-244.

11. Tse GM, Kung FY, Chan AB, Law BK, Chang AR, Lo KW: Clonal analysis of bilateral mammary carcinomas by clinical evaluation and partial allelotyping. Am J Clin Pathol 2003, 120:168-174.

12. Saad RS1, Denning KL, Finkelstein SD, Liu Y, Pereira TC, Lin X, Silverman JF: Diagnostic and prognostic utility of molecular markers in synchronous bilateral breast carcinoma. Mod Pathol 2008, 21:1200-1207.

13. Khan A: The many questions that surround multicentric and multifocal breast cancer. Breast J 2010, 16:219-221.

14. Rosen's Breast Pathology. 2nd edition. Philadelphia: Lippincott Williams \& Wilkins; 2001.

15. Sardanelli F, Giuseppetti GM, Panizza P, Bazzocchi M, Fausto A, Simonetti G, Lattanzio V, Del Maschio A: Italian Trial for Breast MR in Multifocal/ Multicentric Cancer. Sensitivity of MRI versus mammography for detecting foci of multifocal, multicentric breast cancer in fatty and dense breasts using the whole-breast pathologic examination as a gold standard. Am J Roentgenol 2004, 183:1149-1157.

16. Coombs NJ, Boyages J: Multifocal and multicentric breast cancer: does each focus matter? J Clin Oncol 2005, 23:7497-7502.

17. Verkooijen HM1, Chatelain V, Fioretta G, Vlastos G, Rapiti E, Sappino AP, Bouchardy C, Chappuis PO: Survival after bilateral breast cancer: results from a population-based study. Breast Cancer Res Treat 2007, 105:347-357.

18. Carmichael AR, Bendall S, Lockerbie L, Prescott R, Bates T: The long-term outcome of synchronous bilateral breast cancer is worse than metachronous or unilateral tumours. EJSO 2002, 28:388-391.

19. Shi $Y X$, Xia $Q$, Peng RJ, Yuan $Z Y$, Wang SS, An X, Cao $Y$, Tan $Y T$, Jin $Y$, Cai $X Y$, Sun $Y L$, Teng $X Y$, Liu DG, Jiang WQ: Comparison of clinicopathological characteristics and prognoses between bilateral and unilateral breast cancer. J Cancer Res Clin Oncol 2012, 138(4):705-714.

20. McCaul K: Bilateral breast cancer incidence and survival, PhD thesis. University of Adelaide, Faculty of Health Sciences; 2006.

21. Roder D, de Silva P, Zorbas HM, Kollias J, Malycha PL, Pyke CM, Campbell ID: Survival from synchronous bilateral breast cancer: The experience of surgeons participating in the breast audit of the society of breast surgeons of Australia and New Zealand. Asian Pacific J Cancer Prev 2012, 13:1413-1418.

22. Rezo A, Dahlstrom J, Shadbolt B, Rodins K, Zhang Y, Davis AJ: Tumor size and survival in multicentric and multifocal breast cancer. Breast 2011, 20:259-263.

23. Ozmen V, Ozcinar B, Karanlik H, Cabioglu N, Tukenmez M, Disci R, Ozmen T, Igci A, Muslumanoglu M, Kecer M, Soran A: Breast cancer risk factors in Turkish women - a University Hospital based nested case control study. World J Surg Oncol 2009, 7:37

24. Intra M, Rotmensz N, Viale G, Mariani L, Bonanni B, Mastropasqua MG, Galimberti V, Gennari R, Veronesi P, Colleoni M, Tousimis E, Galli A, Goldhirsch A, Veronesi U: Clinicopathologic characteristics of 143 patients with synchronous bilateral invasive breast carcinomas treated in a single institution. Cancer 2004, 101(5):905-912.

25. Ustaalioglu BO, Bilici A, Kefeli U, Seker M, Oncel M, Gezen C, Gumus M, Demirelli F: The importance of multifocal/multicentric tumor on the disease-free survival of breast cancer patients single center experience. Am J Clin Oncol 2012, 35:580-586.

26. Yerushalmi R, Kennecke H, Woods R, Olivotto IA, Speers C, Gelmon KA: Does multicentric/multifocal breast cancer differ from unifocal breast cancer? An analysis of survival and contralateral breast cancer incidence. Breast Cancer Res Treat 2009, 117:365-370.

27. Kuo WH, Yen AM, Lee PH, Chen KM, Wang J, Chang KJ, Chen TH, Tsau HS: Cumulative survival in early-onset unilateral and bilateral breast cancer: an analysis of 1907 Taiwanese women. Br J Cancer 2009, 100:563-570.

28. Cabioglu N, Ozmen V, Kaya H, Tuzlali S, Igci A, Muslumanoglu M, Kecer M, Dagoglu T: Increased lymph node positivity in multifocal and multicentric breast cancer. J Am Coll Surg 2009, 208:67-74.

29. Lebris A, Vildé A, Marret H, Body G, Ouldamer L: Role of imaging procedures in the diagnosis of synchronous bilateral breast cancer. Gynecol Obstet Fertil 2014, 42:14-19. 
30. Ergul N, Kadioglu H, Yildiz S, Yucel SB, Gucin Z, Erdogan EB, Aydin M, Muslumanoglu M: Assessment of multifocality and axillary nodal involvement in early-stage breast cancer patients using 18F-FDG PET/CT compared to contrast-enhanced and diffusion-weighted magnetic resonance imaging and sentinel node biopsy. Acta Radiol 2014. pii: 0284185114539786 . [Epub ahead of print]

31. Engin K: Prognostic factors in bilateral breast cancer. Neoplasma 1994, 41:353-357.

32. Gülay H, Hamaloğlu E, Bulut $\mathrm{O}$, Göksel HA: Bilateral breast carcinoma: 28 years' experience. World J Surg 1990, 14:529-534.

doi:10.1186/1477-7819-12-266

Cite this article as: Kadioğlu et al.: Comparison of the histopathology and prognosis of bilateral versus unilateral multifocal multicentric breast cancers. World Journal of Surgical Oncology 2014 12:266.

\section{Submit your next manuscript to BioMed Central and take full advantage of:}

- Convenient online submission

- Thorough peer review

- No space constraints or color figure charges

- Immediate publication on acceptance

- Inclusion in PubMed, CAS, Scopus and Google Scholar

- Research which is freely available for redistribution 